Groups Geom. Dyn. 4 (2010), 597-603

DOI $10.4171 / \mathrm{GGD} / 96$
Groups, Geometry, and Dynamics

(C) European Mathematical Society

\title{
Special groups with an elementary hierarchy are virtually free-by- $\mathbb{Z}$
}

\author{
Mark Hagen and Daniel T. Wise*
}

\begin{abstract}
Let $G$ be a group with a hierarchy whose edge groups at each level are cyclic. We show that when $G$ is also word-hyperbolic, then $G$ is virtually free-by-cyclic. In particular, this holds for groups splitting as graphs of free groups with cyclic edge groups unless they have Baumslag-Solitar subgroups that are not virtually products.
\end{abstract}

Mathematics Subject Classification (2010). 20E26, 20E06, 20F67.

Keywords. Free-by-cyclic groups, limit groups, right-angled Artin groups, hierarchies.

\section{Introduction}

Roughly speaking, an elementary hierarchy for a group $G$ is a decomposition of $G$ into trivial groups by a finite sequence of splittings over cyclic subgroups (see Definition 2.1). A group is virtually special if it has a finite-index subgroup that embeds in a right-angled Artin group.

Theorem A. Let $G$ be a finitely generated virtually special group with an elementary hierarchy. Then $G$ is virtually free-by- $\mathbb{Z}$.

We note that for general $G$, the kernel $F$ of the $\mathbb{Z}$ quotient given by Theorem A is an infinitely generated free group. In fact, $F$ is finitely generated if and only if $\chi(G)=0$, by a theorem of Feighn and Handel [FH99]. Theorem A applies in the case where $G$ has an elementary hierarchy and is hyperbolic relative to virtually abelian subgroups [Wis]. In particular we obtain:

Corollary B. Every hyperbolic limit group is virtually free-by-ZZ.

Indeed, this holds for limit groups that are hyperbolic relative to a collection of $\mathbb{Z}^{2}$ subgroups. The elementary hierarchy follows from the decomposition result of Kharlampovich-Myasnikov [KM98] and also follows from Sela's "retractive tower"

\footnotetext{
* Research supported by a grant from NSERC.
} 
description of limit groups [Sel03]. Note that $\mathbb{Z}^{3}$ is a limit group that is not free-by$\mathbb{Z}$. The role that right-angled Artin groups play in the proof of Corollary 1 can be sidestepped somewhat by noting that the collection $\mathcal{E}$ of edge groups of the hierarchy survives in some free quotient. It is then not difficult to obtain a $\mathbb{Z}$ quotient with free kernel. We also note that Wilton's theorem, that all finitely generated subgroups of limit groups are virtual retracts [Wil08], gives an alternative way of avoiding the use of right-angled Artin groups. We suspect that hyperbolic limit groups are already free-by- $\mathbb{Z}$, and it is unnecessary to pass to a finite index subgroup. For general limit groups, following the proof of Theorem 3.4 and considering the induced hierarchy of the kernel leads to the conclusion that they are virtually $F$-by- $\mathbb{Z}$, where $F$ is the free product of free abelian groups. We wonder if there is a direct proof that hyperbolic limit groups are virtually free-by- $\mathbb{Z}$.

The simplest non-free examples of groups with an elementary hierarchy are finite graphs of free groups with cyclic edge groups. These include cyclically-pinched onerelator groups as well as $\mathrm{HNN}$ extensions of free groups along cyclic subgroups. In [BFMT09], Baumslag, Fine, Miller and Troeger show that many of the latter two types of group are virtually free-by-cyclic. Our second application completely characterizes when such groups are virtually free-by- $\mathbb{Z}$. Let $\mathrm{BS}(m, n) \cong\left\langle b, s \mid s^{-1} b^{m} s=b^{n}\right\rangle$, where $m, n$ are nonzero integers.

Corollary C. Let $G$ split as a finite graph of free groups with cyclic edge groups. Suppose that $G$ has no $\mathrm{BS}(m, n)$ subgroup with $m \neq \pm n$. Then $G$ is virtually free-by- $\mathbb{Z}$.

Both Corollary 1 and Corollary 1 generalize the well-known fact that surface groups are (virtually) free-by-cyclic. Moreover, they are free-by- $\mathbb{Z}$ in any direction in a sense upon which we elaborate in Remark 3.6.

We note that there are free-by- $\mathbb{Z}$ groups that do not have elementary hierarchies. For example, the fundamental group of a finite-volume cusped hyperbolic 3-manifold $M$ fibering over $S^{1}$ has no codimension-1 subgroup isomorphic to $\mathbb{Z}$. Indeed, one can see this because its JSJ decomposition is trivial. There are word-hyperbolic free-by- $\mathbb{Z}$ such examples as well. Also, while the free product of free-by- $\mathbb{Z}$ groups is readily seen to be free-by- $\mathbb{Z}$, the class of (virtually) free-by-cyclic groups is not closed under HNN extensions along $\mathbb{Z}$ subgroups. The case of amalgamated free products along infinite cyclic subgroups is discussed in Remark 3.6.

Acknowledgement. We thank the referee for helpful corrections.

\section{Elementary hierarchies}

Definition 2.1 (Elementary hierarchy). A group is elementary if it is either trivial or infinite cyclic. The trivial group has an elementary hierarchy of length 0 . Suppose that the group $G$ splits as a connected graph of groups in which each vertex group 
has an elementary hierarchy of length $(n-1)$ and each edge group is elementary. Then $G$ has an elementary hierarchy of length $n$. The group $G$ is said to have an elementary hierarchy if it has an elementary hierarchy of length $n$ for some $n \geq 0$. More generally, $G$ has a hierarchy if it decomposes as above, but with no stipulations about the edge groups.

Formally, a hierarchy of length $n$ for $G$ is a graded set $L=\bigsqcup_{i=1}^{n} L_{i}$, where the $i^{\text {th }}$ level $L_{i}$ is a set of connected graphs of groups, each of which is a decomposition of a vertex group of an element of $L_{i-1}$. The vertex groups of the elements of $L_{n}$ are trivial, and $L_{1}$ is a one-element set containing the initial decomposition of $G$.

A hierarchy for a group $G$ is of finite type if the elements of each level are finite graphs of groups. By definition, the class of groups admitting a (finite-type) elementary hierarchy is closed under (finite) sequences of successive HNN extensions and amalgams over elementary subgroups.

Remark 2.2 (Induced elementary hierarchies). Let $G$ be a group with an elementary hierarchy of length $n$ and let $G^{\prime} \leq G$. Then $G^{\prime}$ has an induced elementary hierarchy of length $n$. Indeed, let $L=\coprod_{i=0}^{n-1} L_{i}$ be an elementary hierarchy for $G^{\prime}$. Let $\widetilde{\Gamma}$ be the Bass-Serre tree corresponding to the single element of $L_{0}=\{\Gamma\}$. Then the action of $G^{\prime}$ on $\widetilde{\Gamma}$ yields a decomposition of $G^{\prime}$ as a graph $\Gamma^{\prime}$ of groups whose vertex groups and edge groups are conjugate into appropriate vertex groups and edge groups of $\Gamma$. Since the vertex groups of the elements of $L_{n}$ are trivial, inductive application of this procedure terminates, yielding an elementary hierarchy $L^{\prime}$ for $G^{\prime}$. In general, the levels of $L^{\prime}$ are infinite and might contain many trees of trivial groups. However, if $G$ has an elementary hierarchy of finite type and $G^{\prime}$ has finite index in $G$, then the induced elementary hierarchy is of finite type. Note that $G^{\prime}$ may have other hierarchies of different length.

Example 2.3 (Graphs of free groups with $\mathbb{Z}$ edge groups). Observe that finitely generated groups that split as graphs of free groups with infinite cyclic edge groups are exactly the groups having finite-type elementary hierarchies of length two.

Lemma 2.4. Let $K$ be a group with an elementary hierarchy (not necessarily of finite type). If the edge groups at each level are trivial, then $K$ is free.

Proof. This is proved by induction on the length $n$ of the hierarchy. The base case $n=0$ simply states that trivial groups are free. For $n \geq 1, K$ splits as a graph of groups whose vertex groups are free by the induction hypothesis, and whose edge groups are trivial. Thus $K$ is a free product of free groups.

Remark 2.5. Suppose that $G$ has an elementary hierarchy of finite type. Then $G$ has a hierarchy in which the underlying graphs of all of the elements of each level are graphs with a single edge. Thus $G$ can be decomposed into trivial groups with a finite sequence of HNN extensions and amalgamated free products along trivial and infinite cyclic subgroups. 
In particular, if $G$ has an elementary hierarchy of finite type, then since each cyclic subgroup is quasi-isometrically embedded, it has a "quasiconvex cyclic hierarchy" in the sense of [Wis]. In the word-hyperbolic case, this implies that $G$ is virtually special.

The Baumslag-Solitar group BS $(m, n)$ has an elementary hierarchy of length 2 when $m, n \neq 0$, since it is a single HNN extension of an infinite cyclic group by an elementary subgroup. However, the edge group is not quasiconvex, and for $m \neq \pm n$, the group $\mathrm{BS}(m, n)$ is not virtually special. This is the reason for the exclusion of such subgroups from Corollary C.

\section{Virtually special groups with elementary finite-type hierarchies}

3.1. Elementary subgroups of right-angled Artin groups. We use the following well-known fact [Gre90].

Lemma 3.1. A finitely-generated right-angled Artin group $R$ has a finite-type hierarchy such that the elements of each level are loops and the vertex groups of the elements at each level are right-angled Artin groups.

Proof. Let $\Gamma$ be the finite graph associated $R$, so that $R \cong\left\langle v \in \Gamma^{0}\right|[u, v]:(u, v) \in$ $\left.\Gamma^{1}\right\rangle$. Let $v$ be a generator. Consider the graph $\ddot{\Gamma}$ obtained by removing the vertex $v$ and all of its incident open edges. Let $\Gamma_{v} \subset \Gamma$ be the full subgraph of $\ddot{\Gamma}$ generated by the vertices adjacent to $v$. Note that for the corresponding right-angled Artin groups we have: $R_{v} \leq \ddot{R}<R$ and $R_{v} \leq Z_{R}(v)$. Accordingly, $R$ splits as an HNN extension of $\ddot{R}$, conjugating the subgroup $R_{v}$ to itself by the stable letter $v$. Since $\ddot{R}$ is generated by a proper subset of the generators of $R_{v}$, the resulting sequence of HNN extensions yields a finite-type hierarchy.

A subgroup $H \leq G$ is a virtual retract if there is a finite-index subgroup $G^{\prime} \leq G$ and a retraction $G^{\prime} \rightarrow G^{\prime} \cap H$. For instance, by Marshall Hall's theorem, every infinite cyclic subgroup of a free group is a virtual retract [Sta83].

Lemma 3.2. Let $r \in R$ be an element of a right-angled Artin group. Then $\langle r\rangle$ is a virtual retract.

Proof. This follows by induction on the length of the hierarchy for $R$ given by Lemma 3.1. In the length- 0 case, $R$ is a trivial group and the result is immediate.

Suppose that the hierarchy for $R$ has length $n$, and that the result holds for any right-angled Artin group with a finite-type hierarchy of length at most $n-1$. By Lemma 3.1, $R$ is an HNN extension $R=\ddot{R} *_{R_{t}}$. For some conjugate $r^{g}$ of $r$, either $r^{g} \in \ddot{R}$, or by the Normal Form Theorem $r^{g}=s$, where $s=r_{0} t^{\epsilon_{0}} r_{1} t^{\epsilon_{1}} \ldots r_{m} t^{\epsilon_{m}}$, each $\epsilon_{i}= \pm 1$ and $r_{i} \in \ddot{R}$ but $r_{i} \notin R_{t}$ whenever $\epsilon_{i-1}=-\epsilon_{i}$. 
In the former case, $r$ is conjugate into $\ddot{R}$, so that $\left\langle r^{g}\right\rangle$ is a virtual retract by the induction hypothesis. Thus $\left\langle r^{g}\right\rangle$ is a virtual retract of $\ddot{R}^{g}$, which itself is a retract of $R$. In the latter case, since the edge group $R_{t}$ is separable, there is a finite quotient $\ddot{R} \rightarrow \bar{R}$ such that $\bar{r}_{i} \notin \bar{R}_{t}$ for each relevant $i$ above. Thus the normal form $\bar{s}$ is nontrivial in the quotient $\ddot{R} *_{R_{t}} \rightarrow \bar{R} * \bar{R}_{t}$.

This quotient group $Q$ is virtually free since it is a finite graph of finite groups [KPS73]. Therefore the infinite cyclic subgroup $\langle\bar{s}\rangle$ is a virtual retract. A finite-index subgroup $Q^{\prime}$ of $Q$ that retracts to $\left\langle\bar{s}^{d}\right\rangle$ induces a finite-index subgroup $R^{\prime}$ of $R$ that retracts to $\left\langle s^{d}\right\rangle$.

Remark 3.3 (Maximal abelian subgroups). Any finitely generated abelian subgroup $A \leq R$ is a virtual retract. Indeed, if $a_{1}, \ldots, a_{m}$ generate $A$, then by Lemma 3.2, there are finite-index subgroups $R_{i} \leq R$ and retractions $\rho_{i}: R_{i} \rightarrow R_{i} \cap\left\langle a_{i}\right\rangle$. There is thus a finite quotient $R \rightarrow \bar{R}$ whose kernel $R^{\prime}$ retracts onto its intersection with each direct factor of $A$, so that $R^{\prime}$ retracts onto $R^{\prime} \cap A$. In particular, every finitely generated abelian subgroup of a right-angled Artin group is a virtual retract.

3.2. Cyclic quotients of special groups. A (finitely-generated) right-angled Artin group is $\pi_{1}$ of a (compact) nonpositively curved cube complex. $G$ is special if it is the fundamental group of a nonpositively curved cube complex that admits a local isometry to the cube complex of a right-angled Artin group [HW08]. Equivalently, $G$ is special if it embeds in some right-angled Artin group. Note that if $\mathrm{G}$ is a finitely generated special group, then it embeds in a finitely generated right-angled Artin group.

Theorem 3.4. Let $G$ be a finitely-generated special group, and let $\&$ be a finite collection of elementary subgroups of $G$. Then there is a finite-index subgroup $\bar{G}$ and a homomorphism $\psi: \bar{G} \rightarrow \mathbb{Z}$ such that $\operatorname{ker}(\psi) \cap g E g^{-1}=\{1\}$ for each $E \in \mathcal{E}$ and $g \in \bar{G}$.

Proof. Let $G$ embed in the finitely generated right-angled Artin group $R$. Then each $E \in \mathcal{E}$ is an elementary subgroup of $R$. Thus it suffices to construct a $\mathbb{Z}$ quotient of some finite-index subgroup of $R$ in which each element of $\mathcal{E}$ virtually embeds. There are finitely many such subgroups. Lemma 3.2 implies that each $E \in \mathcal{E}$ is a retract of an associated finite-index subgroup of $R$. There is thus a finite-index subgroup $R^{\prime}$ such that, for each $E \in \mathcal{E}$, the elementary subgroup $E^{\prime}=E \cap R^{\prime} \leq R^{\prime}$ is a retract. Consequently, each map $H_{1}\left(E^{\prime}\right) \rightarrow H_{1}\left(R^{\prime}\right)$ induced by inclusion is injective.

Hence $H_{1}\left(R^{\prime}\right)$ has a generating set containing a maximal linearly independent set $\left[e_{1}\right],\left[e_{2}\right], \ldots,\left[e_{n}\right]$ of images of homology classes of the generators of the various $E^{\prime}$. Consider a map $\phi: H_{1}\left(R^{\prime}\right) \rightarrow \mathbb{Z}$ constructed by sending all generators to 0 , except for $\left[e_{i}\right] \mapsto z_{i} \neq 0$ for $1 \leq i \leq n$. For each $E^{\prime}$, the kernel of $\phi$ intersects $E^{\prime}$ (and its conjugates) nontrivially only if $\left(z_{i}\right)_{1 \leq i \leq n}$ lies in one of finitely many codimension-1 hyperplanes of $\mathbb{Q}^{n}$. Hence $\phi$ may be chosen so that each $E$ survives in the quotient. 
The subgroups $E^{\prime}$ are abelian, so there is an induced homomorphism $R^{\prime} \rightarrow \mathbb{Z}$ whose kernel does not intersect any conjugate of $E^{\prime}$ nontrivially. Finally, the restriction of this map to $G^{\prime}=R^{\prime} \cap G$ gives the desired map $\psi$.

We now restate and prove Theorem A:

Corollary 3.5. Let $G$ be a virtually special group with an elementary hierarchy of finite type. Then $G$ is virtually free-by- $\mathbb{Z}$.

Proof. Since it has a finite-type hierarchy, $G$ is finitely generated. It is sufficient to handle the case when $G$ is actually special. By Theorem 3.4, there exists a finite-index subgroup $\bar{G} \leq G$ and an epimorphism $\psi: \bar{G} \rightarrow \mathbb{Z}$ such that $\operatorname{ker}(\psi) \cap g E g^{-1}=\{1\}$ for each edge group $E$ and each $g \in \bar{G}$, since there are finitely many edge groups, all elementary. By construction, the elements of each level of the induced hierarchy for $\operatorname{ker}(\psi)$ are graphs of groups with trivial edge groups. Lemma 2.4 implies that $\psi$ has free kernel, and thus $\bar{G}$ is free-by- $\mathbb{Z}$.

Remark 3.6. Consider an amalgam $G=G_{1} *_{H} G_{2}$, where $H$ is infinite cyclic and each $G_{i}$ is free-by- $\mathbb{Z}$. A homomorphism $G \rightarrow \mathbb{Z}$ determines homomorphisms $G_{i} \rightarrow \mathbb{Z}$ that must coincide on $H$. These homomorphisms may differ from those provided by the free-by- $\mathbb{Z}$ assumption, and so it is not clear that the class of freeby- $\mathbb{Z}$ groups is closed under amalgams along $\mathbb{Z}$ subgroups. Roughly, in order to be free-by- $\mathbb{Z}$, each $G_{i}$ must admit enough cyclic quotients with free kernel so that a compatible pair of such maps can be chosen.

For instance, a surface group can be written as an amalgam of two free groups along a $\mathbb{Z}$ subgroup, so that any $\mathbb{Z}$ quotient of either factor has free kernel, and thus any compatible pair gives a quotient with the same property. In this sense, surface groups are free-by- $\mathbb{Z}$ in any "direction". The induced hierarchy for the kernel of these quotients is not of finite type when the genus is at least 2, and in particular the kernel is then an infinitely generated free group. More generally, the proof of Theorem 3.4 uses the elementary hierarchy to specify the choice of "direction", and the induced hierarchy considerations in Corollary 3.5 guarantee that (a finite-index subgroup) of the group is free-by- $\mathbb{Z}$ in that direction.

We now restate and prove Corollary C:

Corollary 3.7. Let $G$ split as a finite graph of free groups with cyclic edge groups. Suppose that $G$ has no $\mathrm{BS}(m, n)$ subgroup with $m \neq \pm n$. Then $G$ is virtually free-by-ZZ

Proof. It was proven in [HW] that $G$ is virtually the fundamental group of a compact special cube complex unless $G$ contains a $\mathrm{BS}(m, n)$, where $m \neq \pm n$. Conversely, no such $\operatorname{BS}(m, n)$ is virtually free-by- $\mathbb{Z}$. Indeed, when neither of $m, n= \pm 1$, then $\mathrm{BS}(m, n)$ is not residually finite and therefore not free-by- $\mathbb{Z}$ [Bau71]. In the case 
$\mathrm{BS}( \pm 1, n)$, all of finite-index subgroups have commutator subgroup that is locally cyclic but not cyclic when $|n|>1$.

\section{References}

[Bau71] G. Baumslag, Finitely generated cyclic extensions of free groups are residually finite. Bull. Austral. Math. Soc. 5 (1971), 87-94. Zbl 0216.08301 MR 0311776

[BFMT09] G. Baumslag, B. Fine, C. F. Miller, III, and D. Troeger, Virtual properties of cyclically pinched one-relator groups. Internat. J. Algebra Comput. 19 (2009), 213-227. Zbl 05572763 MR 2512551

[FH99] M. Feighn and M. Handel, Mapping tori of free group automorphisms are coherent. Ann. of Math. (2) 149 (1999), 1061-1077. Zbl 0938.20022 MR 1709311

[Gre90] E. R. Green, Graph products of groups. PhD thesis, University of Leeds, Leeds 1990.

[HW08] F. Haglund and D. T. Wise, Special cube complexes. Geom. Funct. Anal. 17 (2008), 1551-1620. Zbl 1155.53025 MR 2377497

[HW] T. Hsu and D. T. Wise, Cubulating graphs of free groups with cyclic edge groups. Amer. J. Math., to appear.

[KPS73] A. Karrass, A. Pietrowski, and D. Solitar, Finite and infinite cyclic extensions of free groups. J. Austral. Math. Soc. 16 (1973), 458-466. Zbl 0299.20024 MR 0349850

[KM98] O. Kharlampovich and A. Myasnikov, Irreducible affine varieties over a free group. II. Systems in triangular quasi-quadratic form and description of residually free groups. J. Algebra 200 (1998), 517-570. Zbl 0904.20017 MR 1610664

[Sel03] Z. Sela, Diophantine geometry over groups II: Completions, closures and formal solutions. Israel J. Math. 134 (2003), 173-254. Zbl 1028.20028 MR 1972179

[Sta83] J. R. Stallings, Topology of finite graphs. Invent. Math. 71 (1983), 551-565. Zbl 0521.20013 MR 695906

[Wil08] H. Wilton, Hall's theorem for limit groups. Geom. Funct. Anal. 18 (2008), 271-303. Zbl 1158.20020 MR 2399104

[Wis] D. T. Wise, Research announcement: the structure of groups with a quasiconvex hierarchy. Electron. Res. Announc. Math. Sci. 16 (2009), 44-55. Zbl 1183.20043 MR 2558631

Received October 16, 2009; revised October 26, 2009

M. Hagen, Department of Mathematics and Statistics, McGill University, Montreal, Quebec, Canada H3A 2K6

E-mail: mhagen@math.mcgill.ca

D. T. Wise, Department of Mathematics and Statistics, McGill University, Montreal, Quebec, Canada H3A 2K6

E-mail: wise@math.mcgill.ca 